\title{
The Association of High Prevalence of Trophozoites in Peripheral Blood with Lower Antibody Response to $P$. falciparum Infected Erythrocytes among Asymptomatic Children in Sudan
}

\author{
Sara N. Mohamed, ${ }^{1}$ Dina A. Hassan, ${ }^{1}$ Abdelrahim M. El Hussein, ${ }^{1}$ Ihssan M. Osman, \\ Muntasir E. Ibrahim, ${ }^{3}$ Hiba S. Mohamed, ${ }^{3}$ Bakri Y. Nour, ${ }^{4}$ and Nasreldin H. Abdulhadi ${ }^{5}$ \\ ${ }^{1}$ Central Laboratory, Ministry of Higher Education and Scientific Research, Khartoum, Sudan \\ ${ }^{2}$ Faculty of Medicine, Al-Zaiem Al Azhari University, Khartoum, Sudan \\ ${ }^{3}$ Institute of Endemic Diseases, University of Khartoum, 11111 Khartoum, Sudan \\ ${ }^{4}$ The Blue Nile Institute for Training and Research, Gezira University, Wad Madani, Sudan \\ ${ }^{5}$ College of Pharmacy, The National Ribat University, Khartoum, Sudan
}

Correspondence should be addressed to Nasreldin H. Abdulhadi; nasralhadi@gmail.com

Received 12 March 2016; Accepted 1 June 2016

Academic Editor: Christophe Chevillard

Copyright (C) 2016 Sara N. Mohamed et al. This is an open access article distributed under the Creative Commons Attribution License, which permits unrestricted use, distribution, and reproduction in any medium, provided the original work is properly cited.

\begin{abstract}
Background. The most prominent variant surface antigens (VSAs) of Plasmodium falciparum are the var gene-encoded Plasmodium falciparum erythrocyte membrane protein 1 (PfEMP1) family, which serves as a parasite-sequestering ligand to endothelial cells. In this study we have examined the antibody reactivity of autologous plasma from symptomatic and asymptomatic malaria infected children against the infected erythrocytes' surface antigens using flow cytometry. Methods. Ethidium-bromide-labelled erythrocytic mature forms of $P$. falciparum parasites obtained from symptomatic and asymptomatic children were sequentially incubated with autologous plasma and fluorescein isothiocyanate-conjugated (FITC) antihuman IgG. Plasma antibody reactivity was detected by flow cytometry. Results. Asymptomatic children had more prevalence of trophozoites in peripheral blood (66\%) compared to symptomatic children (16\%), $p=0.002$. The mean percentage of infected RBCs reacting with autologous sera was 89.78 among symptomatic children compared to 79.62 among asymptomatic children $(p=0.09)$. Moreover, the mean fluorescence intensity (MFI) in the asymptomatic was significantly higher compared to symptomatic children ( $p$ value $=0.040)$. Conclusion. Variant surface antigens on Plasmodium falciparum infected RBCs from symptomatic malaria children tend to be better recognized by IgG antibodies. This may suggest a role of some IgG antibodies in severity of malaria.
\end{abstract}

\section{Background}

The variant surface antigens (VSAs) including the most recognized Plasmodium falciparum erythrocyte membrane protein 1 (PfEMP1) are responsible for the pathogenicity of malaria infection. Through interaction with host molecules such as ICAM1, CD36, CR1, and CD31, VSAs play a central role in mediating cytoadherence of infected erythrocytes to host cells. This is believed to be responsible for the severe pathology associated with P. falciparum malaria [1]. Parasite-infected erythrocytes from young children and those with severe malaria were shown to be better recognized by antibodies from semi-immune children than those from older children or those with nonsevere malaria $[2,3]$. Levels of antibodies to many malaria antigens may vary with the seasonality of malaria transmission, often being higher during periods of high malaria transmission than at the end of a low transmission season [4-8]. Antibodies to malaria antigens often tend to be higher in individuals who are infected at the time their antibodies are measured than in noninfected individuals $[2,9,10]$. The predominance of $\operatorname{IgG} 1$ and $\operatorname{IgG} 3$ cytophilic antibodies in endemic areas has been associated 
with either lower parasitaemia [11] or a lower risk of malaria attack $[12,13]$. Noncytophilic antibodies, such as IgG4, may inhibit effector mechanisms by competing with cytophilic antibodies and are considered nonprotective [14, 15]. IgG2 is noncytophilic but could be correlated with protection in individuals carrying a specific allelic variant of monocytes Fc $\gamma$ RIIA receptor that can bind IgG2 [16]. Most antibody studies use laboratory strains as source of antigens which may not reflect antigen repertoire of field strains. This preliminary study is intended to measure total plasma IgG antibody response to VSA antigens using autologous plasma among symptomatic and asymptomatic children. The levels of antibodies so determined were then correlated to the reduced iRBCs cytoadherence phenomenon, previously reported by our group as a possible determinant of asymptomatic malaria among Fallata tribe children in Sudan [17].

\section{Subjects and Methods}

The study was approved by the Ethical Committee of the Institute of Endemic Diseases, University of Khartoum, Sudan. It was conducted in Al Keraiba, a suburb of Wad Madani, central Sudan, during November-December 2009, at the peak of malaria transmission in this area. A total of 55 children, belonging to the Fallata tribe, of whom 24 presented with fever with or without other symptoms of malaria and 31 were asymptomatic, are included in this study. P. falciparum infection was confirmed by ICT test and microscopy. Three $\mathrm{mL}$ venous blood sample was obtained in citrated vacutainer tube from each child after obtaining signed consent forms from their parents. The symptomatic children were obtained from Al Keraiba health center while the asymptomatic children were obtained during a crosssectional survey for malaria in Al Keraiba primary school. Asymptomatic children were followed up for 48 hours and 8 of them who developed symptoms within the follow-up period were excluded.

2.1. Parasitaemia. Levels of initial parasitaemia (parasites/ $\mu \mathrm{L}$ ) were determined by counting parasites against 200 leukocytes in the thick smears taken at the time of sampling assuming that each subject had 8000 leukocytes/ $\mu \mathrm{L}$.

2.2. Parasite Maturation and Fixation. Infected erythrocytes were obtained from each sample, washed in RPMI medium, and grown in culture for 48-60 hours [18]. Trophozoites/schizonts so obtained were enriched by gel floatation [18] and preserved in transfix solution (Transfix ${ }^{\circledR}$ Cytomark Ltd., Buckingham, England).

2.3. Flow Cytometry. Trophozoite/schizont infected RBCs (iRBCs) obtained after gel floatation and fixation were incubated with 1:200 diluted autologous plasma in FACS staining buffer (FSB) for 45 minutes at room temperature. Samples were washed twice with FSB and incubated with fluorescein-conjugated antihuman IgG (Sigma, UK) and $2 \mu \mathrm{g} / \mathrm{mL}$ ethidium bromide at room temperature for 30 minutes. Samples were then washed twice with FBS.
Coulter EPICS XL-MCL flow cytometer was used for acquirement and analysis of 10000 events/sample. Populations of parasitized erythrocytes expressing antiserum-reactive VSA (EtdBr- and plasma antibodies-positive) were gated. The mean fluorescence intensities (MFI) were determined for symptomatic and asymptomatic groups.

2.4. Statistics. Statistical significance was calculated by twosample, two-tailed Student's $t$-test (SPSS Software, version 13.0). Comparisons were considered statistically significant at $p<0.05$.

\section{Results}

There were no significant differences between symptomatic and asymptomatic children in terms of age; however the mean starting parasitaemia was significantly higher among symptomatic children $(4.59 \pm 0.52$ parasite/ $\mu \mathrm{L})$ compared to asymptomatic children $(1.33 \pm 0.52$ parasite/ $\mu \mathrm{L})$. All symptomatic and asymptomatic samples gave positive antibody response after counting for the background given by noninfected RBCs. The mean $( \pm$ SEM) percentage of infected RBCs reacting with autologous sera (VSA-positive cells) was $89.78 \pm 1.67$ among symptomatic children compared to $79.62 \pm 2.97$ among asymptomatic children $(p=0.09)$. The mean fluorescence intensity, MFI ( \pm SEM), in the symptomatic $(32.65 \pm 10.3)$ was significantly higher compared to asymptomatic children $(8.2 \pm 1.0)(p$ value $=0.04$, Table 1$)$.

\section{Discussion}

Previously we have reported an in vivo reduced sequestration of infected erythrocytes among asymptomatic children in the suburbs of Wad Madani, the same area of the present study [17]. It is well known that the major antigenic ligands found to be responsible for the cytoadhesive properties of the infected red blood cells (iRBCs) are members of the P. falciparum erythrocyte membrane protein 1 (PfEMP1) family. These variable surface antigens (VSAs) are placed on knob-like structures on the surface of the iRBCs and bind to different host vascular adhesins, including CD36, ICAM1, VCAM1, and CSA [19]. We assumed a differential antibody response to the VSA of the iRBCs that might block or mediate cytoadhesion leading to asymptomatic or symptomatic disease, respectively. However, levels of antibodies to many malaria antigens may vary with the seasonality of malaria transmission [7]. Antibodies often tend to be higher in individuals who also have malaria parasites at the time their antibodies are measured than in those without parasites [2]. Moreover, follow-up studies intended to define protective immune responses against malaria are usually handicapped. This is attributable to a difficulty in distinguishing between individuals who do not get clinical episodes because they were unexposed and those who are truly immune. We assume that having the infection at the time of blood collection and using autologous plasma to look at antibody response may overcome the exposure heterogeneity and brevity of antibody response as a measure of immunity [20]. In this study, 
TABLE 1: Age, parasitaemia, prevalence of trophozoites, \% VSA, and MFI.

\begin{tabular}{lcccc}
\hline Group & $\begin{array}{c}\text { Age (year) } \\
\mathrm{M} \pm \text { SEM }\end{array}$ & $\begin{array}{c}\text { Parasitaemia } \\
(\text { parasites } / \mu \mathrm{L})^{*} \\
\mathrm{M} \pm \mathrm{SEM}\end{array}$ & $\begin{array}{c}\text { Prevalence of } \\
\text { trophozoites }(\%)\end{array}$ & $\begin{array}{c}\text { MFI VSA } \\
\mathrm{M} \pm \mathrm{SEM}\end{array}$ \\
\hline Symptomatic $(N=24)$ & $8.5 \pm 2$ & $4.59 \pm 0.52$ & 16 & $89.78 \pm 1.6$ \\
Asymptomatic $(N=23)$ & $8.8 \pm 0.6$ & $1.33 \pm 0.45$ & 66 & $79.62 \pm 2.9$ \\
$p$ value & 0.990 & 0.027 & 0.002 & 0.090 \\
\hline
\end{tabular}

${ }^{*} \times 10^{3}$.

the lower antibody response among asymptomatic children which was accompanied with reduced cytoadherence, as evident by higher percentage of circulating trophozoites, may suggest that some antibodies can mediate cytoadhesion and lead to immune responses that manifest as clinical presentation. Alternatively, the symptomatic group may also represent cases of very early symptomatic infections in nonimmune children. Our results showed clearly a quantitative difference of total IgG antibody response between symptomatic and asymptomatic children. These results may encourage further detailed qualitative studies of subclasses of antibodies developed during malaria infection to show their possible role in clinical protection in this population.

\section{Conclusion}

The preliminary finding in this study of reduced antibody response to $P$. falciparum malaria among clinically protected children and its association with the reduced cytoadhesion suggests a role for antibodies in sequestration. This hypothesis may be tested by further longitudinal studies to investigate the possible role of different classes and subclasses of immunoglobulins and the effects of age, season, and frequency of infections.

\section{Competing Interests}

The authors declare that there is no conflict of interests regarding the publication of this paper.

\section{Authors' Contributions}

Sara N. Mohamed carried out parasite isolates culturing and preparation for flow cytometry. Dina A. Hassan contributed to the conception and design of the study. Abdelrahim M. El Hussein participated in the designing, coordination, and acquisition of funding. Ihssan M. Osman calibrated the flow cytometry method. Muntasir E. Ibrahim and Hiba S. Mohamed participated in acquisition of funding for field work and in revising the paper. Bakri Y. Nour coordinated and provided facilities for field work. Nasreldin $\mathrm{H}$. Abdulhadi conceived of the study and prepared the draft of the paper.

\section{Acknowledgments}

The authors sincerely thank the children and parents for their cooperation. They thank Mr. Khalid Ali Sidig, Central
Laboratory, Khartoum, Sudan, for helping in the collection of samples and blood films preparation and Mr. Osama Ali Mohamed El-Tayeb from Radiation and Isotopes Centre for his great help in manoeuvring the flow cytometer software. This work has been partially funded by the Ministry of Higher Education, Sudan. Finally, the authors would like to commemorate Sara N. Mohamed who had a remarkable contribution to this work, but unfortunately she has passed away during the publication process.

\section{References}

[1] S. Kyes, P. Horrocks, and C. Newbold, "Antigenic variation at the infected red cell surface in malaria," Annual Review of Microbiology, vol. 55, pp. 673-707, 2001.

[2] P. C. Bull, M. Kortok, O. Kai et al., "Plasmodium falciparuminfected erythrocytes: agglutination by diverse Kenyan plasma is associated with severe disease and young host age," Journal of Infectious Diseases, vol. 182, no. 1, pp. 252-259, 2000.

[3] M. A. Nielsen, T. Staalsoe, J. A. L. Kurtzhals et al., "Plasmodium falciparum variant surface antigen expression varies between isolates causing severe and nonsevere malaria and is modified by acquired immunity," The Journal of Immunology, vol. 168, no. 7, pp. 3444-3450, 2002.

[4] J. A. Vande Waa, J. B. Jensen, M. A. S. Akood, and R. Bayoumi, "Longitudinal study on the in vitro immune response to Plasmodium falciparum in Sudan," Infection and Immunity, vol. 45, no. 2, pp. 505-510, 1984.

[5] K. Fruh, O. Doumbo, H.-M. Muller et al., "Human antibody response to the major merozoite surface antigen of Plasmodium falciparum is strain specific and short-lived," Infection and Immunity, vol. 59, no. 4, pp. 1319-1324, 1991.

[6] D. R. Cavanagh, I. M. Elhassan, C. Roper et al., "A longitudinal study of type-specific antibody responses to Plasmodium falciparum merozoite surface protein-1 in an area of unstable malaria in Sudan," The Journal of Immunology, vol. 161, no. 1, pp. 347-359, 1998.

[7] H. A. Giha, T. G. Theander, T. Staalsø et al., "Seasonal variation in agglutination of Plasmodium falciparum-infected erythrocytes," The American Journal of Tropical Medicine and Hygiene, vol. 58, no. 4, pp. 399-405, 1998.

[8] I. Nebie, A. B. Tiono, D. A. Diallo et al., "Do antibody responses to malaria vaccine candidates influenced by the level of malaria transmission protect from malaria?" Tropical Medicine and International Health, vol. 13, no. 2, pp. 229-237, 2008.

[9] F. Al-Yaman, B. Genton, M. Falk et al., "Humoral response to Plasmodium falciparum ring-infected erythrocyte surface antigen in a highly endemic area of Papua New Guinea," American Journal of Tropical Medicine and Hygiene, vol. 52, no. 1, pp. 66-71, 1995. 
[10] S. M. Kinyanjui, T. Mwangi, P. C. Bull, C. I. Newbold, and K. Marsh, "Protection against clinical malaria by heterologous immunoglobulin $G$ antibodies against malaria-infected erythrocyte variant surface antigens requires interaction with asymptomatic infections," Journal of Infectious Diseases, vol. 190, no. 9, pp. 1527-1533, 2004.

[11] Y. P. Shi, U. Sayed, S. H. Qari et al., "Natural immune response to the C-terminal 19-kilodalton domain of Plasmodium falciparum merozoite surface protein 1," Infection and Immunity, vol. 64, no. 7, pp. 2716-2723, 1996.

[12] P. Tangteerawatana, S. Krudsood, K. Chalermrut, S. Looareesuwan, and S. Khusmith, "Natural human IgG subclass antibodies to Plasmodium falciparum blood stage antigens and their relation to malaria resistance in an endemic area of Thailand Southeast," The Southeast Asian Journal of Tropical Medicine and Public Health, vol. 32, no. 2, pp. 247-254, 2001.

[13] J.-L. Sarthou, G. Angel, G. Aribot et al., "Prognostic value of anti-Plasmodium falciparum-specific immunoglobulin G3, cytokines, and their soluble receptors in West African patients with severe malaria," Infection and Immunity, vol. 65, no. 8, pp. 3271-3276, 1997.

[14] O. Garraud, S. Mahanty, and R. Perraut, "Malaria-specific antibody subclasses in immune individuals: a key source of information for vaccine design," Trends in Immunology, vol. 24, no. 1, pp. 30-35, 2003.

[15] P. A. M. Warmerdam, J. G. J. Van de Winkel, A. Vlug, N. A. C. Westerdaal, and P. J. A. Capel, "A single amino acid in the second Ig-like domain of the human Fc $\gamma$ receptor II is critical for human IgG2 binding," The Journal of Immunology, vol. 147, no. 4, pp. 1338-1343, 1991.

[16] C. Aucan, Y. Traoré, F. Tall et al., "High immunoglobulin G2 (IgG2) and low IgG4 levels are associated with human resistance to Plasmodium falciparum malaria," Infection and Immunity, vol. 68, no. 3, pp. 1252-1258, 2000.

[17] D. A. Hassan, A. P. Arez, H. S. Mohamed, A. M. Elhussein, M. E. Ibrahim, and N. H. Abdulhadi, "The reduced sequestration of Plasmodium-falciparum-infected erythrocytes among malaria cases with sickle-cell trait: In-vivo evidence from Sudan," Annals of Tropical Medicine and Parasitology, vol. 102, no. 8, pp. 743-748, 2008.

[18] W. Trager and J. B. Jensen, "Human malaria parasites in continuous culture," Science, vol. 193, no. 4254, pp. 673-675, 1976.

[19] S. M. Kraemer and J. D. Smith, "A family affair: var genes, PfEMP1 binding, and malaria disease," Current Opinion in Microbiology, vol. 9, no. 4, pp. 374-380, 2006.

[20] S. M. Kinyanjui, P. Bejon, F. H. Osier, P. C. Bull, and K. Marsh, "What you see is not what you get: implications of the brevity of antibody responses to malaria antigens and transmission heterogeneity in longitudinal studies of malaria immunity," Malaria Journal, vol. 8, article 242, 2009. 


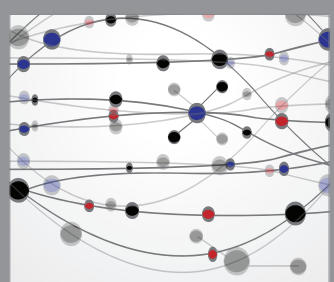

The Scientific World Journal
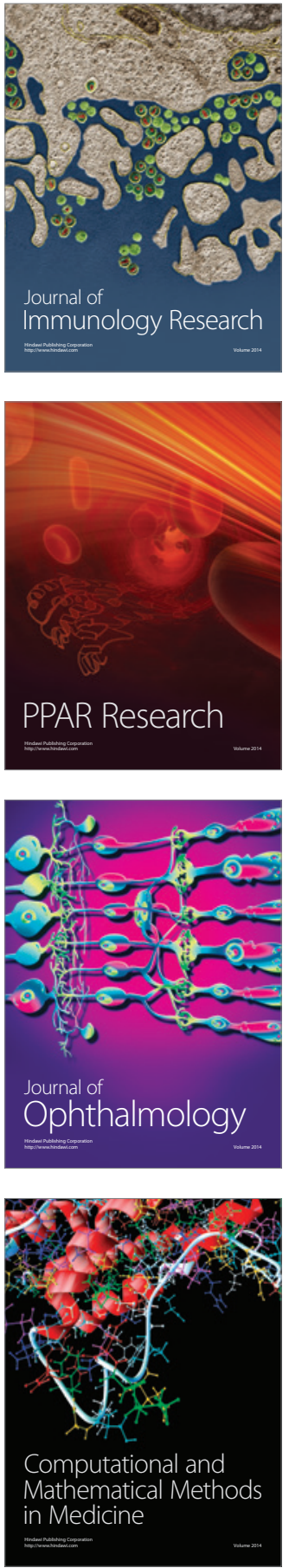

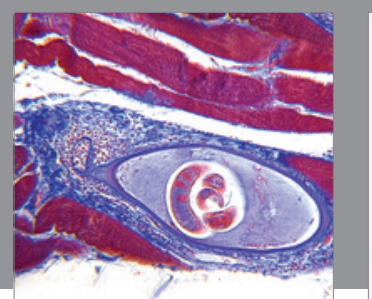

Gastroenterology Research and Practice

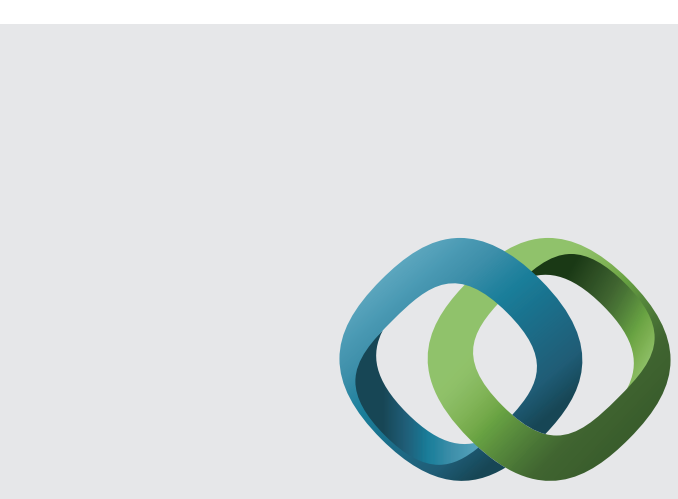

\section{Hindawi}

Submit your manuscripts at

http://www.hindawi.com
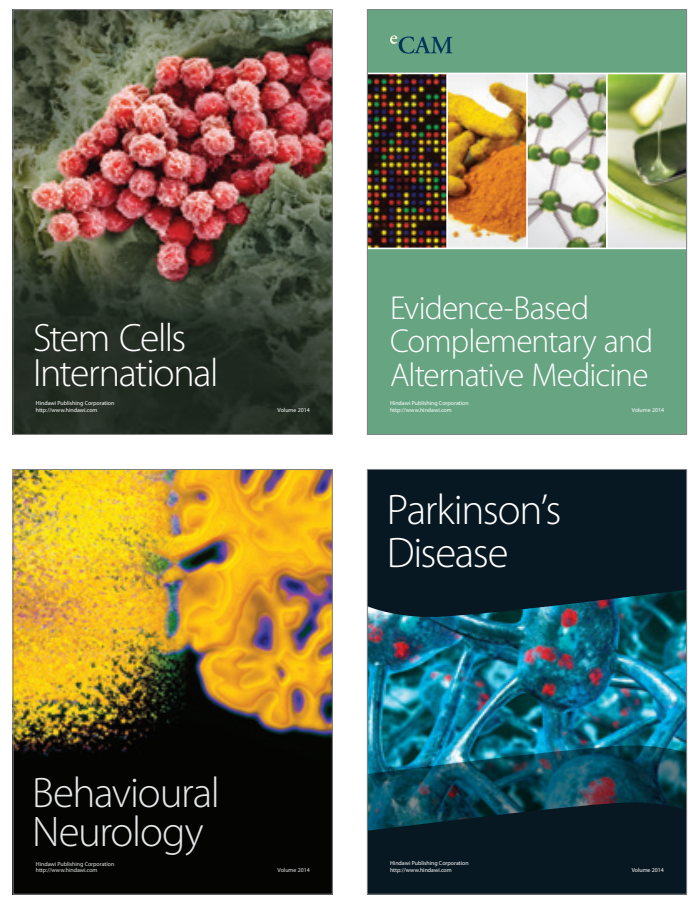
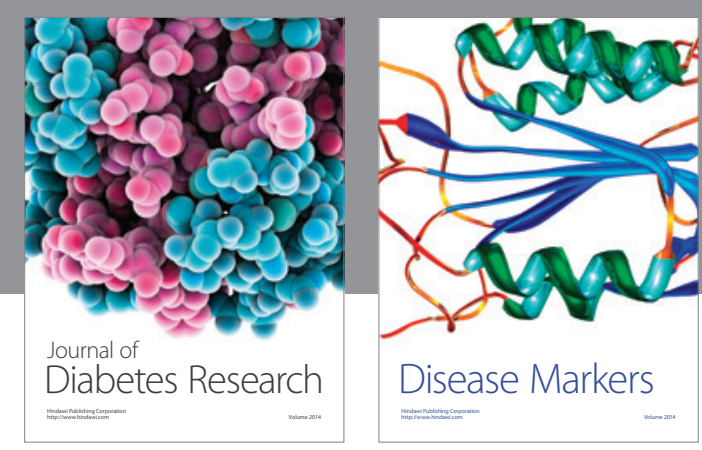

Disease Markers
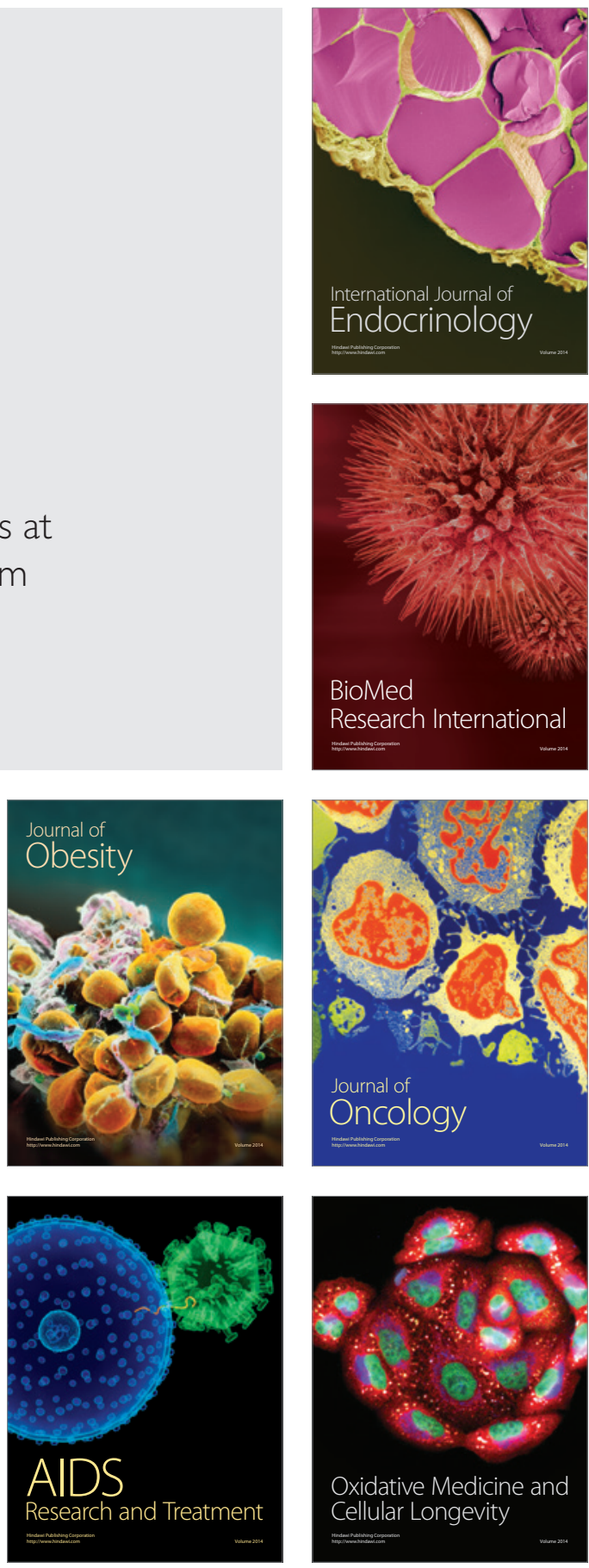